\title{
Decursin inhibits UVB-induced MMP expression in human dermal fibroblasts via regulation of nuclear factor- $\kappa B$
}

\author{
BO-MI HWANG ${ }^{1 *}$, EUN-MI NOH ${ }^{*}$, JONG-SUK KIM $^{1}$, JEONG-MI KIM $^{1}$, JIN-KI HWANG $^{2}$, \\ HYE -KYUNG KIM ${ }^{3}$, JAE-SEON KANG ${ }^{4}$, DO-SUNG KIM ${ }^{5}$, HAN-JUNG CHAE ${ }^{5}$, \\ YONG-OUK YOU ${ }^{6}$, KANG-BEOM KWON ${ }^{2}$ and YOUNG-RAE LEE ${ }^{2,6}$ \\ ${ }^{1}$ Department of Biochemistry, Institute of Medical Science, Chonbuk National University Medical School, Jeonju 560-182; \\ ${ }^{2}$ Center for Metabolic Function Regulation, Wonkwang University School of Medicine, Iksan 570-749; \\ ${ }^{3}$ Department of Urology, Medical School, and Institute for Medical Sciences, Chonbuk National University, Jeonju 561-712; \\ ${ }^{4}$ Department of Pharmacy, Kyungsung University, Busan 608-736; ${ }^{5}$ Department of Pharmacology, \\ School of Medicine, Chonbuk National University, Jeonju 560-182; ${ }^{6}$ Department of Oral Biochemistry, \\ School of Dentistry, Wonkwang University, Iksan 570-749, Republic of Korea
}

Received July 25, 2012; Accepted September 17, 2012

DOI: $10.3892 /$ ijmm.2012.1202

\begin{abstract}
Decursin, a coumarin compound, was originally isolated from the roots of Angelica gigas almost four decades ago, and it was found to exhibit cytotoxicity against various types of human cancer cells and anti-amnesic activity in vivo through the inhibition of AChE activity. However, the antiskin photoaging effects of decursin have not been reported to date. In the present study, we investigated the inhibitory effects of decursin on the expression of matrix metalloproteinase (MMP)-1 and MMP-3 in human dermal fibroblast (HDF) cells. Western blot analysis and real-time PCR revealed that decursin inhibited the ultraviolet (UV)B-induced expression of MMP-1 and MMP-3 in a dose-dependent manner. Decursin significantly blocked the UVB-induced activation of nuclear factor- $\kappa \mathrm{B}(\mathrm{NF}-\kappa \mathrm{B})$. However, decursin showed no effect on MAPK or AP-1 activity. In this study, decursin prevented the UVB-induced expression of MMPs via the inhibition of $\mathrm{NF}-\kappa \mathrm{B}$ activation. In conclusion, decursin may be a potential agent for the prevention and treatment of skin photoaging.
\end{abstract}

\section{Introduction}

Skin aging can be divided into intrinsic (chronologic) aging, which is the process of senescence that affects all body

Correspondence to: Professor Young-Rae Lee or Professor Kang-Beom Kwon, Center for Metabolic Function Regulation, Wonkwang University School of Medicine, Iksan 570-749, Republic of Korea

E-mail: mindyr@wku.ac.kr

E-mail: desson@wku.ac.kr

*Contributed equally

Key words: decursin, matrix metalloproteinase, ultraviolet B, nuclear factor- $\kappa \mathrm{B}$, skin photoaging organs and extrinsic aging (photoaging), which occurs due to exposure to environmental factors. An important factor that influences extrinsic aging is sunlight, particularly exposure to ultraviolet (UV)B irradiation, which causes photoaging. Chronic exposure of human skin to UVB radiation results in photoaging and induces the production of matrix metalloproteinases (MMPs) (1).

MMPs are responsible for the degradation of the collagenous extracellular matrix (ECM) in connective tissues (2). MMP-1 preferentially degrades fibrillar collagens, which maintain the tensile strength of fetal membranes. In contrast, MMP-3 degrades an extremely wide array of ECM substrates and can activate secreted zymogenic forms of other MMPs (3).

UV exposure is an important factor in photoaging. UV irradiation of cultured human dermal fibroblasts (HDFs) in vitro or human skin in vivo induces the production of MMPs (4-6). Excessive matrix degradation by UV-induced MMPs secreted by various types of cells (e.g., keratinocytes, fibroblasts and inflammatory cells) has been shown to contribute significantly to connective tissue damage that occurs during photoaging $(7,8)$. UVB irradiation can induce MMP expression by activating transcription factors, such as nuclear factor- $\kappa \mathrm{B}(\mathrm{NF}-\kappa \mathrm{B})$ and activator protein-1 (AP-1) $(9,10)$. The mitogen-activated protein kinase (MAPK) signaling pathway is important for AP-1 activation; I $\mathrm{B}$ kinase (IKK), phosphoinositide 3 kinase (PI3K)-Akt and p38 MAPK have been shown to activate $N F-\kappa B$, depending on the cell type $(11,12)$. Thus, the inhibition of UVB-induced MMP expression and/or its upstream regulatory pathways is critical for the treatment of photoaging of the skin.

Decursin is a coumarin compound found in the roots of Angelica gigas Nakai, which has been traditionally used in Korean folk medicine as a tonic and for the treatment of anemia and other diseases (13). Decursin induces cell cycle arrest and apoptosis in human prostate, breast, bladder and colon cancer cells (14-16). Recent reports have demonstrated 
that decursin blocks MMP-9 expression through the inhibition of $\mathrm{NF}-\kappa \mathrm{B}$ activation in macrophages and cancer cells (17-19). However, the inhibitory effects of UVB-induced MMP expression through $\mathrm{NF}-\kappa \mathrm{B}$ activation by decursin are not yet well defined.

In the present study, we evaluated the preventive effects of decursin on the UVB-induced production of MMPs in HDFs. Decursin blocked the UVB-induced NF- $\mathrm{B}$ pathway, which inhibits the expression of MMPs. These results suggest that decursin is useful for the prevention of skin photoaging.

\section{Materials and methods}

Materials. 3-(4,5-Dimethyl-thiazol-2-yl)-2,5-diphenyltetrazolium bromide (MTT), dimethyl sulfoxide (DMSO) and anti- $\beta$-actin antibody were purchased from Sigma (St. Louis, MO, USA). Primary antibodies for MMP-1 and MMP-3 were obtained from R\&D Systems (Minneapolis, MN, USA). Dulbecco's modified Eagle's medium (DMEM) with high glucose level, Medium 154, growth supplement, fetal bovine serum (FBS) and phosphate-buffered saline (PBS) were obtained from Gibco-BRL (Gaithersburg, ME, USA). Primary antibodies for $\mathrm{p} 50, \mathrm{p} 65, \mathrm{I} \kappa \mathrm{B} \alpha$, proliferating cell nuclear antigen (PCNA) and horseradish peroxidase (HRP)conjugated IgG were obtained from Santa Cruz Biotechnology, Inc. (Santa Cruz, CA, USA).

Plant extracts and purification. The roots of A. gigas Nakai (Umbelliferae family) were extracted serially with methanol, ethylacetate and n-butanol and fractionated. From the ethylacetate fraction, decursin was isolated using silica gel column chromatography. After column chromatography, the structure of the purified coumarin compounds, decursin $\left(\mathrm{C}_{19} \mathrm{H}_{20} \mathrm{O}_{5}\right)$ and decursinol angelate (molecular weight, $328 \mathrm{~g}$ ) were characterized by gas chromatography (Shimadzu, Kyoto, Japan), nuclear magnetic resonance (JEOL JNM-LA 400; Japan) and mass spectroscopy (JEOL-AX 505WA) at Daegu Haany University, Daegu, Korea.

Isolation and culture of HDFs. HDFs were aseptically isolated from foreskin. The epidermis and dermis were separated by incubation in media with $0.9 \mathrm{U} / \mathrm{ml}$ dispase at $4^{\circ} \mathrm{C}$ for $16 \mathrm{~h}$. After the epidermis and dermis were mechanically separated, the dermis was minced, attached to the surface of a tissue culture flask and incubated with DMEM containing $10 \%$ FBS for 1-2 weeks (20). Dermal fibroblasts that spread as radial outgrowths from the attached pieces of dermis were cultured in DMEM containing $10 \%$ FBS and $1 \%$ antibiotics at $37^{\circ} \mathrm{C}$ in a $5 \% \mathrm{CO}_{2}$ incubator.

UV irradiation. HDFs were rinsed twice with $\mathrm{PBS}$ and irradiated using a UVB cross-linker $(6 \times 8 \mathrm{~W}, 312 \mathrm{~nm}$; Model CL-508M; Vilber Lourmat, Paris, France) (20). HEKn were irradiated using a Stratalinker UV crosslinker (Model 2400; Agilent Technologies, Cold Spring, NY, USA). Immediately after irradiation, fresh serum-free medium was added to the HDFs, and complete growth medium was added to the HEKn. Responses were measured after incubation for each experimental condition. The same schedule of medium changes was followed for control cells.

Determination of cell viability. The protective effect of decursin against UV-induced cytotoxicity of HDFs was determined using the MTT assay. Briefly, HDFs were seeded at a density of $3 \times 10^{4}$ cells/plate and allowed to attach. After $24 \mathrm{~h}$, the cells were treated with various concentrations of decursin $(1,5,10,30$ and $50 \mu \mathrm{M})$. After incubation for $24 \mathrm{~h}$, the cells were washed twice with PBS and MTT $(0.5 \mathrm{mg} / \mathrm{ml} \mathrm{PBS})$ was added to each well. The plates were incubated at $37^{\circ} \mathrm{C}$ for $30 \mathrm{~min}$. Formazan crystals that had formed were dissolved by adding DMSO (100 $\mu \mathrm{l} /$ well) and the absorbance was measured at $570 \mathrm{~nm}$ using a microplate reader (Model 3550; Bio-Rad, Richmond, CA, USA).

Trypan blue exclusion test for cytotoxicity. Cells were seeded onto a $10-\mathrm{cm}$ dish and allowed to attach for $24 \mathrm{~h}$. They were then treated with UVB at $25 \mathrm{~mJ} / \mathrm{cm}^{2}$. After $24 \mathrm{~h}$, the cells were detached from the wells by treatment with trypsin, followed by staining with trypan blue; non stained cells were counted under an optical microscope with a hemocytometer.

Western blot analysis. HDFs $\left(2 \times 10^{6}\right.$ cells) were irradiated with UVB ( 25 or $15 \mathrm{~mJ} / \mathrm{cm}^{2}$ ); the cells were treated with decursin for $24 \mathrm{~h}$ and lysed using $40 \mu \mathrm{l}$ of ice cold M-PER ${ }^{\circledR}$ Mammalian Protein Extraction Reagent (Pierce Biotechnology, Inc., Rockford, IL, USA). Protein concentrations in the lysates were determined using the Bradford method (21). Samples were separated using $10 \%$ SDS-PAGE gels with 3\% stacking gels; the resolved proteins were transferred to a Hybond ${ }^{\mathrm{TM}}$ PVDF membrane using a western blot apparatus (Bio-Rad). Polyvinylidene fluoride (PVDF) membranes were blotted with $1 \mu \mathrm{g} / \mathrm{ml}$ of primary antibodies for MMP-1, MMP-3, p50, p65, PCNA, or $\beta$-actin. HRP-conjugated IgG was used as a secondary antibody. Protein expression levels were determined by analyzing the signals captured on the PVDF membranes using an image analyzer (LAS-1000; Fuji Film, Japan).

Quantitative real-time PCR assay. Total RNA was extracted from cells using a FastPure ${ }^{\mathrm{TM}}$ RNA kit (Takara Bio, Inc., Shiga, Japan). RNA concentration and purity were determined by measuring the absorbance at both 260 and $280 \mathrm{~nm}$. Then, cDNA was synthesized from $1 \mu \mathrm{g}$ of total RNA using a PrimeScript $^{\mathrm{TM}}$ RT reagent kit (Takara Bio, Inc.). MMP-1 and MMP-3 mRNA expression levels were analyzed using realtime PCR with the ABI PRISM 7900 sequence detection system and the SYBR-Green reagent (Applied Biosystems, Foster City, CA, USA). Primers used in the reaction were:MMP-1 (NM 002424.2) sense,5'-AGTGACTGGGAAACCGATGCTGA-3' and antisense, 5'-CTCTTGGCAAATCTGGCCTGTAA-3'; MMP-3 (NM 002422) sense, 5'-ATTCCATGGAGCCAGGCTTTC-3' and antisense, 5'-CATTTGGGTCAAACTCCAACTGTG-3' and GAPDH (NM 002046) sense, 5'-ATGGAAATCCC ATCACCATCTT-3' and antisense, 5'-CGCCCCACTTGA TTTTGG-3'. To control for variations in the mRNA concentration, all results were normalized to the housekeeping gene GAPDH. Relative 
quantitations were performed using the comparative $\Delta \Delta \mathrm{Ct}$ method according to the manufacturer's instructions.

Determination of MMP-1 and MMP-3 secretion with ELISA. HDFs were seeded in 100-mm culture dishes at a density of $2 \times 10^{6}$ cells/dish and then irradiated with UVB $\left(25 \mathrm{~mJ} / \mathrm{cm}^{2}\right)$. Following $24 \mathrm{~h}$ of incubation, the culture supernatants were collected and centrifuged at $10,000 \times \mathrm{g}$ for $5 \mathrm{~min}$ to remove the particulate matter and stored at $-80^{\circ} \mathrm{C}$ in fresh tubes. The protein concentration in the supernatants was determined using the Bradford method (21). The active MMP-1 in culture supernatants was quantified by fluorescent assay, using the Fluorokine E Human Active MMP-1 Fluorescent assay kit, and MMP-3 in the cell culture supernatants was then determined using Quantikine ELISA kits (all from R\&D Systems), according to the manufacturer's protocol.

Preparation of nuclear extract. HDFs $\left(2 \times 10^{6}\right.$ cells) were irradiated with $25 \mathrm{~mJ} / \mathrm{cm}^{2} \mathrm{UVB}$ and then treated with decursin for $3 \mathrm{~h}$. Cells were immediately washed twice, scraped into $1.5 \mathrm{ml}$ of ice cold $\mathrm{PBS}(\mathrm{pH} 7.9$ ) and then pelleted at $12,000 \mathrm{x} g$ for $30 \mathrm{sec}$. Cytoplasmic and nuclear extracts were prepared from cells using NE-PER ${ }^{\circledR}$ Nuclear and Cytoplasmic Extraction Reagents (Pierce Biotechnology).

Electrophoretic mobility shift assay (EMSA). Activation of $\mathrm{NF}-\kappa \mathrm{B}$ and AP-1 was assayed with a gel mobility shift assay using nuclear extracts. An oligonucleotide containing the $\kappa$-chain ( $\kappa$ B, 5'-CCGGTTAACAGAGGGGGCTTTCCGAG-3') or AP-1 (5'-CGCTTGATGAGTCAGCCGGAA-3') binding site was synthesized and used as a probe for the gel retardation assay. The two complementary strands were annealed and labeled with $[\alpha-32 \mathrm{P}] \mathrm{dCTP}$. Labeled oligonucleotides (10,000 cpm), $10 \mu \mathrm{g}$ of nuclear extracts and binding buffer [10 mM Tris- $\mathrm{HCl}$ (pH 7.6), $500 \mathrm{mM} \mathrm{KCl,} 10 \mathrm{mM}$ EDTA, $50 \%$ glycerol, $100 \mathrm{ng}$ poly $(\mathrm{dI} \cdot \mathrm{dC}), 1 \mathrm{mM}$ dithiothreitol] were then incubated for $30 \mathrm{~min}$ at room temperature in a final volume of $20 \mu \mathrm{l}$. The reaction mixtures were analyzed by electrophoresis on $4 \%$ polyacrylamide gels in $0.5 \mathrm{X}$ Tris-borate buffer. The gels were dried and examined by autoradiography. Specific binding was controlled by competition with a 50-fold excess and cold AP-1 oligonucleotide.

Statistical analysis. Statistical analysis was performed using analysis of variance (ANOVA) and Duncan's test. A P-value $<0.05$ was considered to indicate a statistically significant result.

\section{Results}

Decursin protects HDFs against UVB irradiation. The structure of decursin is shown in Fig. 1A. To investigate the cytotoxicity of decursin, HDFs were treated with various concentrations of decursin for $24 \mathrm{~h}$. Cell viability was determined using the MTT assay. Decursin did not cause a significant change in the viability of HDFs up to $50 \mu \mathrm{M}$ (Fig. 1B). To investigate the cell protective effect of decursin on UVB-induced cytotoxicity, cells were incubated with the indicated concentrations of decursin for $24 \mathrm{~h}$ in the presence of UVB. UVB-induced cytotoxicity was determined using
A
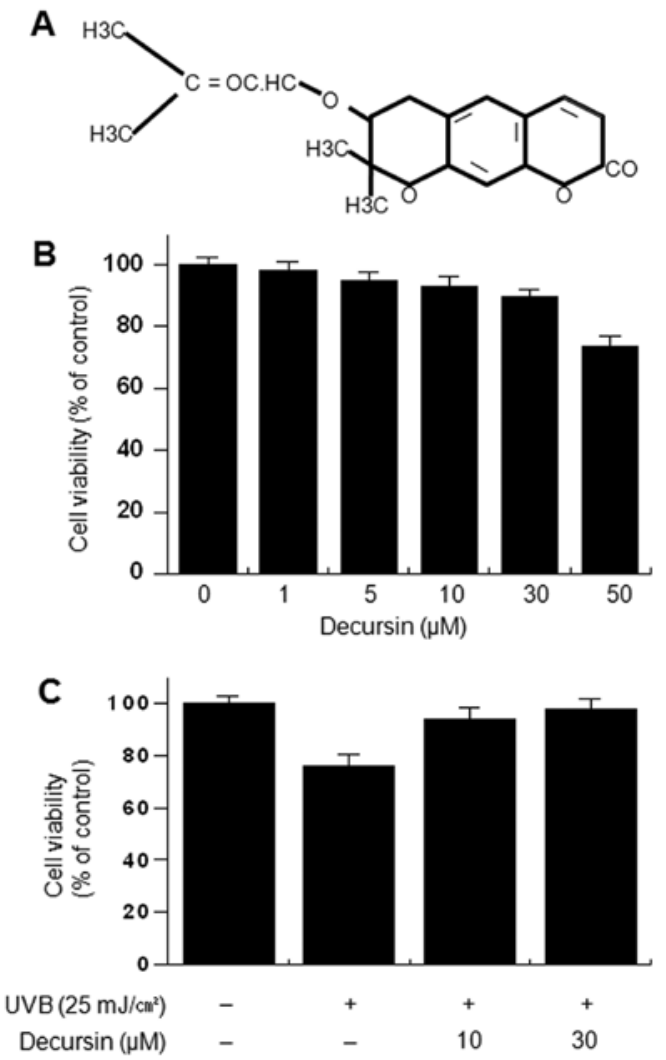

Figure 1. Structure of decursin and the effect of decursin on the viability of HDFs. (A) Chemical structure of decursin. (B) HDFs were cultured in 96-well plates until reaching $70 \%$ confluence, and various concentrations of decursin were added to the cells for $24 \mathrm{~h}$. An MTT assay was used to detect cell viability. (C) Cells were cultured in 100-mm culture dishes until reaching $70 \%$ confluence and incubated with the indicated concentrations of decursin for $24 \mathrm{~h}$ under UVB irradiation. Cell viability was determined by cell counting. The optical density value of control cells was normalized to $100 \%$. Data represent the means \pm SEM of 3 independent experiments.

the trypan blue exclusion test. Decursin (10 and $30 \mu \mathrm{M})$ significantly inhibited cell toxicity induced by UVB irradiation (Fig. 1C).

Decursin inhibits the UVB-induced expression and secretion of MMP-1 and MMP-3 in HDFs. UVB activates MMP secretion, which is a hallmark of skin aging $(4,5,22)$. We examined the effects of decursin on UVB-induced expression of MMP-1 and MMP-3. Western blot analysis revealed that irradiation of HDFs with UVB $\left(25 \mathrm{~mJ} / \mathrm{cm}^{2}\right)$ markedly increased MMP-1 and MMP-3 levels (Fig. 2A). The UVB-induced increase in MMP levels was significantly reduced by treatment with decursin. Consistent with these results, real-time PCR analysis also showed an increase in expression of MMP-1 and MMP-3 mRNA after UVB irradiation while treatment of HDFs with decursin suppressed this UVB-induced increase in MMP-1 and MMP-3 expression (Fig. 2B). We also determined the effect of decursin on UVB-induced MMP secretion with ELISA. UVB irradiation of HDFs resulted in an increase in MMP-1 and MMP-3 secretion, while decursin significantly diminished the UVB-induced MMP-1 and MMP-3 secretion (Fig. 2C). Decursin itself had no effects on expression and secretion of MMP-1 and MMP-3 in HDFs. These results 
A

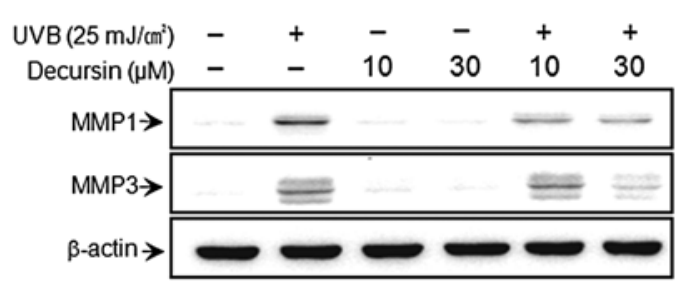

B
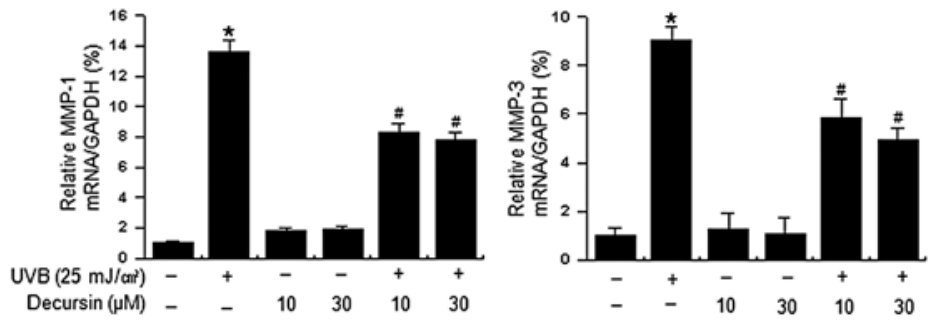

C
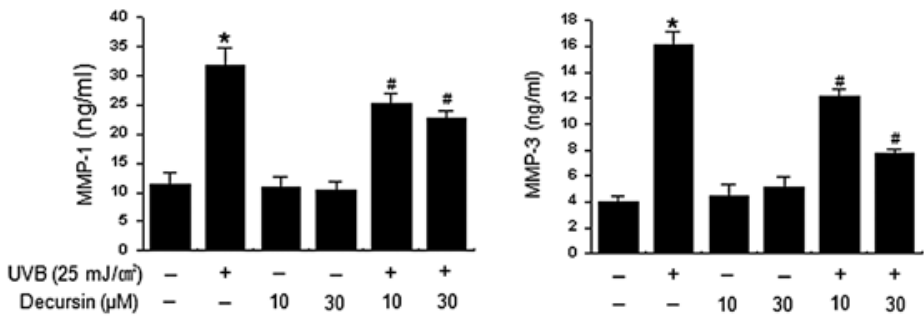

Figure 2. Effect of decursin on UVB-induced MMP-1 and MMP-3 expression in HDFs. Cells were stimulated with UVB (25 mJ/cm $\left.{ }^{2}\right)$ and incubated with the indicated concentrations of decursin for $24 \mathrm{~h}$. (A) Cell lysates were analyzed by western blot analysis with anti-MMP-1 and -MMP-3 antibodies. (B) Total cellular RNA was analyzed using real-time PCR for MMP-1 and MMP-3. (C) The presence of MMP-1 and MMP-3 in cell-free culture supernatants was measured using a commercially available ELISA kit as described in Materials and methods. Each value represents the means \pm SEM of 3 independent experiments. ${ }^{*} \mathrm{P}<0.01$ vs. untreated control; ${ }^{\#} \mathrm{P}<0.01$ vs. UVB.

indicate that decursin inhibits the UVB-induced expression and secretion of MMP-1 and MMP-3 in HDFs.

Effect of decursin on UVB-induced NF- $\kappa B$ and AP-1 DNA-binding activities. To clarify the mechanism of decursinmediated inhibition of MMP-1 and MMP-3 expression, the effect of decursin on UVB-induced activation of NF- $\mathrm{kB}$ and AP-1 was evaluated using EMSA and western blot analysis. As shown in Fig. 3A and B, pre-treatment with decursin inhibited UVB-induced DNA binding activity of NF- $\mathrm{KB}$, but not AP-1. Decursin itself had no effect on the DNA binding activity of NF- $\mathrm{KB}$ or AP-1. Additionally, we determined the levels of p65, p50, p-c-Jun in the nuclear fraction. Cell treatment with UVB resulted in increased levels of p65, p50 and p-c-Jun; however, decursin blocked the UVB-induced translocation of p65 and p50 to the nucleus (Fig. 3C). These results suggest that decursin specifically blocks NF- $\mathrm{kB}$ activation in HDFs. The IкB kinase (IKK) enzyme complex is part of the signal transduction cascade upstream of NF- $\mathrm{kB}$. IKK specifically phosphorylates the inhibitory I $\mathrm{KB}$ protein. Under basal conditions, the cytoplasmic protein I $\kappa$ B directly binds to $\mathrm{p} 65$ and p50 subunits and represses their nuclear translocation. IKK phosphorylation results in the dissociation of I $\mathrm{B}$ from $\mathrm{NF}-\kappa \mathrm{B}$ and thereby activates NF- $\mathrm{B}$ (23-26). Therefore, we determined the changes in the levels of p-IKK $\alpha \beta$ and p-IкB $\alpha$ in the cytoplasmic fraction. The cytoplasmic fraction of UVB-stimulated HDFs showed higher levels of p-IKK $\alpha \beta$ and $\mathrm{p}-\mathrm{I} \kappa \mathrm{B} \alpha$ than in unstimulated cells; however, the UVB-induced increase in the levels of $\mathrm{p}-\mathrm{IKK} \alpha \beta$ and $\mathrm{p}-\mathrm{I} \kappa \mathrm{B} \alpha$ was significantly suppressed by treatment with decursin (Fig. 3D).

Effect of decursin on the UVB-induced MAP kinase signaling pathway. Since MAP kinase is an upstream regulator of $\mathrm{NF}-\mathrm{KB}$ and AP-1, the role of MAP kinase (ERK, p38 and
JNK) in the activation of MMP expression is fairly well understood $(27,28)$. We investigated the effect of decursin on UVB-induced activation of MAP kinase. Decursin showed no effects on MAPK (Fig. 4). These results suggest that the MAPK pathway is not involved in the regulation of UVB-induced expression of MMP by decursin.

\section{Discussion}

In the present study, we demonstrated the preventive effects of decursin on photoaging caused by MMP-1 and MMP-3. In previous studies, decursin was found to prevent MMP-9 expression by suppression of the NF- $\mathrm{BB}$ pathway in cancer cells and macrophages (17-19). Our results also demonstrated that decursin blocked UVB-induced activation of NF- $\mathrm{KB}$, which has an important role in MMP-1 and MMP-3 expression.

Skin aging can be attributed to extrinsic aging (photoaging) and intrinsic (chronological) aging. Photoaging involves premature skin aging caused by repeated exposure to the sun $(8,29,30)$. UV irradiation of cultured HDFs in vitro or human skin in vivo was found to induce the expression of MMP-1 and MMP-3, which play important roles in ECM components during skin aging $(5,6,31)$. Varani et al (32) reported that MMP levels increase and collagen synthesis decreases in sun-protected human skin in vivo as age increases. Moreover, it was suggested that excessive matrix degradation by UV-induced MMPs secreted by various types of cells (e.g., keratinocytes, fibroblasts and inflammatory cells) contributes substantially to connective tissue damage that occurs during skin photoaging $(7,8,33)$. Thus, we focused on the targets of decursin's action in signal transduction pathways involved in the induction of the two major MMP family members after UVB irradiation. 
A

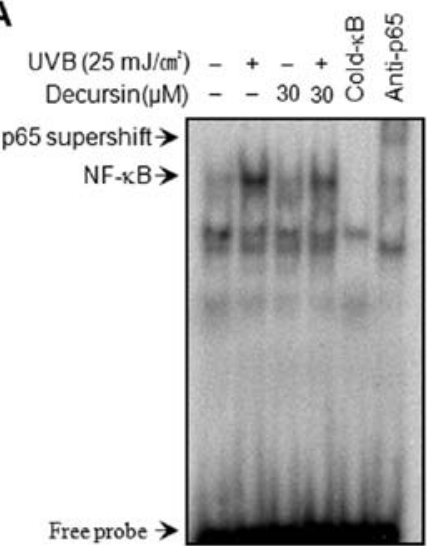

C

D

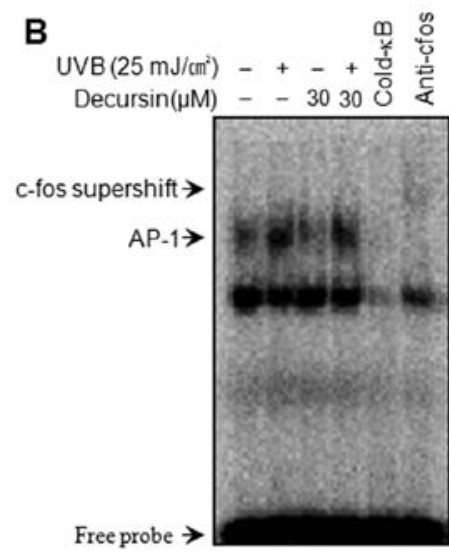

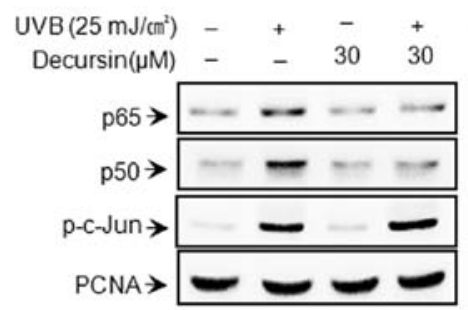

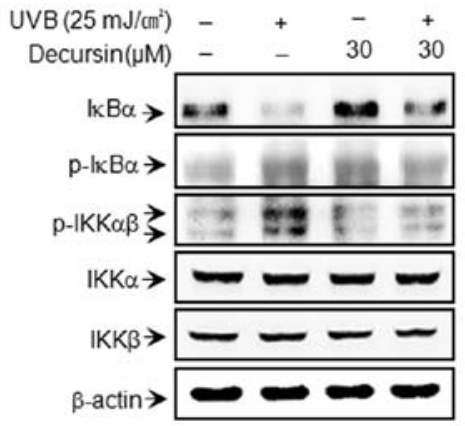

Figure 3. Decursin blocks UVB-induced NF- $\mathrm{BB}$ activation in HDFs. Cells were stimulated with UVB $\left(25 \mathrm{~mJ} / \mathrm{cm}^{2}\right)$ and incubated with the indicated concentrations of decursin. (A and B) Following $3 \mathrm{~h}$ of incubation, nuclear extracts were prepared. NF- $\mathrm{\kappa B}$ and AP-1 DNA binding was analyzed using electrophoretic mobility shift analysis as described in Materials and methods. (C and D) Western blotting was performed to determine the nuclear levels of NF- $\mathrm{BB}$ (p50 and p65) and AP-1 (p-c-Jun) subunits, as well as the cytoplasmic levels of p-IKK $\alpha \beta, \operatorname{IKK} \alpha, \operatorname{IKK} \beta, \mathrm{p}-\mathrm{I \kappa B} \alpha$ and IкB $\alpha$.

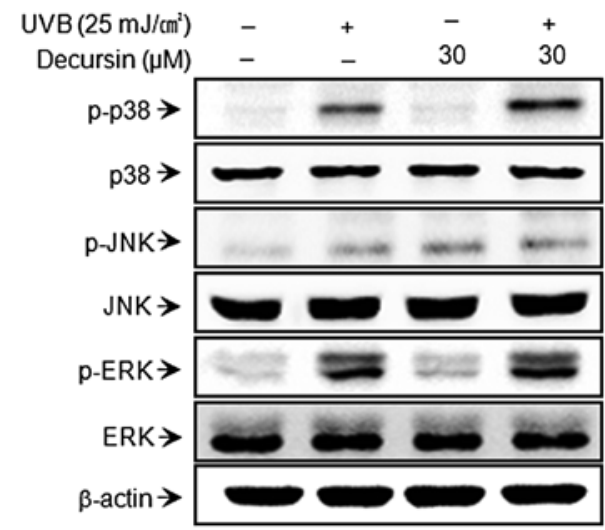

Figure 4. Effect of decursin on UVB-induced MAPK signaling activation in HDFs. Cells were pre-treated with UVB $\left(25 \mathrm{~mJ} / \mathrm{cm}^{2}\right)$ for $15 \mathrm{~min}$ in the presence or absence of decursin. Cell lysates were prepared for western blotting with antibodies against $\mathrm{p} 38$, JNK and ERK, as well as with phospho-specific antibodies for these antigens (p-p38, p-JNK and p-ERK).

UV irradiation includes three types: UVA (wavelength, $320-400 \mathrm{~nm})$, UVB (280-320 nm) and UVC (200-280 nm). In particular, studies concerning skin have focused on UVB intensity due to stratospheric ozone depletion $(34,35)$. It is well known that the UVB-inducible genes involved in skin aging are primarily composed of several MMPs involved in the degradation of the connective tissues of the skin $(22,35)$. Recent studies have focused on the regulatory molecular mechanisms underlying UVB-induced upregulation of MMPs $(36,37)$.

The present study found that transcription factors may be targets of decursin during UV-induced skin damage. NF- $\mathrm{KB}$ and AP-1 are ubiquitous transcription factors that govern the expression of genes encoding cytokines, chemokines, growth factors, cell adhesion molecules and several acute phase proteins in healthy and disease states $(38,39)$. Therefore, the development of strategies that target these transcription factors may provide novel therapeutic tools for treating or preventing various diseases. UVB-mediated photoaging is prevented by the suppression of NF- $\kappa \mathrm{B}$ and AP-1 activation $(31,40,41)$. In fact, NF- $\kappa \mathrm{B}$ and AP-1 are known to increase MMP-1 expression in the dermis $(42,43)$. These studies suggest that NF- $\kappa \mathrm{B}$ and AP-1 play important roles in MMP expression after UV irradiation. Previous studies demonstrated that NF- $\kappa \mathrm{B}$ and AP-1 are molecular targets in decursin-treated cells $(44,45)$, which suggests that targeting NF- $\kappa \mathrm{B}$ and AP-1 in UV irradiation-mediated MMP expression by using decursin may provide a novel therapeutic tool 
for treating or preventing photoaging. Our results showed that decursin strongly blocked UVB-induced NF- $\mathrm{BB}$ activation (Fig. 3). Furthermore, decursin significantly inhibited UVB-induced expression of MMPs in HDFs (Fig. 2). Our data indicate that decursin is a potent inhibitor of UVB-mediated $\mathrm{NF}-\kappa \mathrm{B}$ activation, which blocks the UVB-induced expression of MMPs in HDFs.

The MAPK pathway is involved in the regulation of cell proliferation, apoptosis, cytokine expression and MMP production. The three major MAPK families, JNK, ERK and p38 kinase, are expressed in HDFs, and the active phosphorylated forms can also be detected $(46,47)$. Previous studies have shown that the MAPK signaling pathway is important for AP-1 activation; I- $\kappa$ B kinase (IKK), phosphoinositide 3 kinase (PI3K)-Akt and p38 MAPK have been shown to activate NF- $\mathrm{KB}$, depending on the cell type $(11,12,48-50)$. In this study, decursin displayed no effects on phosphorylation of p38, JNK and ERK. These data indicate that decursin is involved in $\mathrm{NF}-\kappa \mathrm{B}$, but not in the MAPK signaling pathway in HDFs

In conclusion, the development of novel MMP inhibitors may be a promising strategy for skin cancer therapy and photoaging. Our results demonstrate that decursin is a potent inhibitor of UVB-induced expression of MMPs that blocks the NF- $\kappa B$ signaling pathway in HDFs. Therefore, decursin may be a potential therapeutic candidate for the prevention and treatment of photoaging.

\section{Acknowledgements}

This study was supported by the National Research Foundation of Korea (NRF) grant funded by the Korea government (MEST) (nos. 2011-0030716 and 2011-0023921).

\section{References}

1. Ho JN, Lee YH, Park JS, et al: Protective effects of aucubin isolated from Eucommia ulmoides against UVB-induced oxidative stress in human skin fibroblasts. Biol Pharm Bull 28: 1244-1248, 2005.

2. Scharffetter-Kochanek K, Brenneisen $\mathrm{P}$, Wenk J, et al: Photoaging of the skin from phenotype to mechanisms. Exp Gerontol 35: 307-316, 2000.

3. Knauper V, Lopez-Otin C, Smith B, Knight G and Murphy G: Biochemical characterization of human collagenase-3. J Biol Chem 271: 1544-1550, 1996.

4. Pillai S, Oresajo C and Hayward J: Ultraviolet radiation and skin aging: roles of reactive oxygen species, inflammation and protease activation, and strategies for prevention of inflammation-induced matrix degradation - a review. Int J Cosmet Sci 27 17-34, 2005.

5. Brenneisen P, Sies H and Scharffetter-Kochanek K: Ultraviolet-B irradiation and matrix metalloproteinases: from induction via signaling to initial events. Ann NY Acad Sci 973: 31-43, 2002.

6. Rittié L and Fisher GJ: UV-light-induced signal cascades and skin aging. Ageing Res Rev 1: 705-720, 2002.

7. Fisher GJ, Datta SC, Talwar HS, et al: Molecular basis of suninduced premature skin ageing and retinoid antagonism. Nature 379: 335-339, 1996.

8. Chung JH, Seo JY, Lee MK, et al: Ultraviolet modulation of human macrophage metalloelastase in human skin in vivo. J Invest Dermatol 119: 507-512,2002.

9. Cooper SJ and Bowden GT: Ultraviolet B regulation of transcription factor families: roles of nuclear factor-kappa $B$ (NF-kappaB) and activator protein-1 (AP-1) in UVB-induced skin carcinogenesis. Curr Cancer Drug Targets 7: 325-334, 2007.

10. Bell S, Degitz K, Quirling M, Jilg N, Page S and Brand K: Involvement of NF-kappaB signalling in skin physiology and disease. Cell Signal 15: 1-7, 2003.

11. Karin M: The regulation of AP-1 activity by mitogen-activated protein kinases. J Biol Chem 270: 16483-16486, 1995.
12. Madrid LV, Mayo MW, Reuther JY and Baldwin AS Jr.: Akt stimulates the transactivation potential of the RelA/p65 subunit of NF-kappa B through utilization of the Ikappa B kinase and activation of the mitogen-activated protein kinase p38. J Biol Chem 276: 18934-18940, 2001.

13. Ahn Q, Jeong SJ, Lee HJ, et al: Inhibition of cyclooxygenase-2-dependent survivin mediates decursin-induced apoptosis in human KBM-5 myeloid leukemia cells. Cancer Lett 298: 212-221, 2010.

14. Yim D, Singh RP, Agarwal C, Lee S, Chi H and Agarwal R: A novel anticancer agent, decursin, induces G1 arrest and apoptosis in human prostate carcinoma cells. Cancer Res 65: 1035-1044, 2005.

15. Jiang C, Guo J, Wang Z, et al: Decursin and decursinol angelate inhibit estrogen-stimulated and estrogen-independent growth and survival of breast cancer cells. Breast Cancer Res 9: R77, 2007.

16. Kim WJ, Lee SJ, Choi YD and Moon SK: Decursin inhibits growth of human bladder and colon cancer cells via apoptosis, G1-phase cell cycle arrest and extracellular signal-regulated kinase activation. Int J Mol Med 25: 635-641, 2010.

17. Kim WJ, Lee MY, Kim JH, Suk K and Lee WH: Decursinol angelate blocks transmigration and inflammatory activation of cancer cells through inhibition of PI3K, ERK and NF- $\kappa \mathrm{B}$ activation. Cancer Lett 296: 35-42, 2010.

18. Kim JH, Jeong JH, Jeon ST, et al: Decursin inhibits induction of inflammatory mediators by blocking nuclear factor-kappaB activation in macrophages. Mol Pharmacol 69: 1783-1790, 2006.

19. Lee SH, Lee JH, Kim EJ, et al: A novel derivative of decursin, CSL-32, blocks migration and production of inflammatory mediators and modulates PI3K and NF-kappaB activities in HT1080 cells. Cell Biol Int 36: 683-688, 2012.

20. Lee YR, Noh EM, Jeong EY, et al: Cordycepin inhibits UVB-induced matrix metalloproteinase expression by suppressing the NF-kappaB pathway in human dermal fibroblasts. Exp Mol Med 41: 548-554, 2009.

21. Bradford MM: A rapid and sensitive method for the quantitation of microgram quantities of protein utilizing the principle of protein-dye binding. Anal Biochem 72: 248-254, 1976.

22. Fisher GJ, Kang S, Varani J, et al: Mechanisms of photoaging and chronological skin aging. Arch Dermatol 138: 1462-1470, 2002.

23. Jacobs MD and Harrison SC: Structure of an IkappaBalpha/ NF-kappaB complex. Cell 95: 749-758, 1998.

24. Regnier CH, Song HY, Gao X, Goeddel DV, Cao Z and Rothe M: Identification and characterization of an IkappaB kinase. Cell 90: 373-383, 1997.

25. Mercurio F, Zhu H, Murray BW, et al: IKK-1 and IKK-2: cytokine-activated IkappaB kinases essential for NF-kappaB activation. Science 278: 860-866, 1997.

26. Karin M: How NF-kappaB is activated: the role of the IkappaB kinase (IKK) complex. Oncogene 18: 6867-6874, 1999.

27. Chung TW, Moon SK, Chang YC, et al: Novel and therapeutic effect of caffeic acid and caffeic acid phenyl ester on hepatocarcinoma cells: complete regression of hepatoma growth and metastasis by dual mechanism. FASEB J 18: 1670-1681, 2004.

28. Eberhardt W, Huwiler A, Beck KF, Walpen S and Pfeilschifter J: Amplification of IL-1 beta-induced matrix metalloproteinase- 9 expression by superoxide in rat glomerular mesangial cells is mediated by increased activities of NF-kappa B and activating protein-1 and involves activation of the mitogen-activated protein kinase pathways. J Immunol 165: 5788-5797, 2000.

29. Uitto J: Connective tissue biochemistry of the aging dermis. Age-related alterations in collagen and elastin. Dermatol Clin 4: 433-446, 1986.

30. Gilchrest BA: A review of skin ageing and its medical therapy. Br J Dermatol 135: 867-875, 1996.

31. Chung JH, Hanft VN and Kang S: Aging and photoaging. J Am Acad Dermatol 49: 690-697, 2003.

32. Varani J, Perone P, Fligiel SE, Fisher GJ and Voorhees JJ: Inhibition of type I procollagen production in photodamage: correlation between presence of high molecular weight collagen fragments and reduced procollagen synthesis. J Invest Dermatol 119: $122-129,2002$.

33. Chung JH, Seo JY, Choi HR, et al: Modulation of skin collagen metabolism in aged and photoaged human skin in vivo. J Invest Dermatol 117: 1218-1224, 2001.

34. Lloyd RE, Larson RA, Adair TL and Tuveson RW: Cu(II) sensitizes pBR322 plasmid DNA to inactivation by UV-B (280-315 nm). Photochem Photobiol 57: 1011-1017, 1993. 
35. Brenneisen P, Wenk J, Wlaschek M, Krieg T and ScharffetterKochanek K: Activation of p70 ribosomal protein S6 kinase is an essential step in the DNA damage-dependent signaling pathway responsible for the ultraviolet $\mathrm{B}$-mediated increase in interstitial collagenase (MMP-1) and stromelysin-1 (MMP-3) protein levels in human dermal fibroblasts. J Biol Chem 275: 4336-4344, 2000.

36. Kim JK, Mun S, Kim MS, Kim MB, Sa BK and Hwang JK 5,7-Dimethoxyflavone, an activator of PPARalpha/gamma, inhibits UVB-induced MMP expression in human skin fibroblast cells. Exp Dermatol 21: 211-216, 2012.

37. Chiang HM, Chen HC, Lin TJ, Shih IC and Wen KC: Michelia alba extract attenuates UVB-induced expression of matrix metalloproteinases via MAP kinase pathway in human dermal fibroblasts. Food Chem Toxicol 50: 4260-4269, 2012.

38. Bakiri L, Matsuo K, Wisniewska M, Wagner EF and Yaniv M: Promoter specificity and biological activity of tethered AP-1 dimers. Mol Cell Biol 22: 4952-4964, 2002.

39. Chen FE and Ghosh G: Regulation of DNA binding by Rel/ NF-kappaB transcription factors: structural views. Oncogene 18 6845-6852, 1999.

40. Adhami VM, Afaq F and Ahmad N: Suppression of ultraviolet B exposure-mediated activation of NF-kappaB in normal human keratinocytes by resveratrol. Neoplasia 5: 74-82, 2003.

41. Tanaka K, Hasegawa J, Asamitsu K and Okamoto T: Prevention of the ultraviolet B-mediated skin photoaging by a nuclear factor kappaB inhibitor, parthenolide. J Pharmacol Exp Ther 315: 624-630, 2005.

42. Bond M, Baker AH and Newby AC: Nuclear factor kappaB activity is essential for matrix metalloproteinase- 1 and -3 upregulation in rabbit dermal fibroblasts. Biochem Biophys Res Commun 264: 561-567, 1999.
43. Chung JH: Photoaging in Asians. Photodermatol Photoimmunol Photomed 19: 109-121, 2003.

44. Liu Q, Loo WT, Sze SC and Tong Y: Curcumin inhibits cell proliferation of MDA-MB-231 and BT-483 breast cancer cells mediated by down-regulation of NFkappaB, cyclinD and MMP-1 transcription. Phytomedicine 16: 916-922, 2009.

45. Kim SY, Jung SH and Kim HS: Curcumin is a potent broad spectrum inhibitor of matrix metalloproteinase gene expression in human astroglioma cells. Biochem Biophys Res Commun 337: 510-516, 2005.

46. Shim JS, Kwon YY, Han YS and Hwang JK: Inhibitory effect of panduratin A on UV-induced activation of mitogen-activated protein kinases (MAPKs) in dermal fibroblast cells. Planta Med 74: 1446-1450, 2008

47. Bae JY, Choi JS, Choi YJ, et al: (-)Epigallocatechin gallate hampers collagen destruction and collagenase activation in ultraviolet-B-irradiated human dermal fibroblasts: involvement of mitogen-activated protein kinase. Food Chem Toxicol 46: 1298-1307, 2008

48. Yao J, Xiong S, Klos K, et al: Multiple signaling pathways involved in activation of matrix metalloproteinase-9 (MMP-9) by heregulin-betal in human breast cancer cells. Oncogene 20: 8066-8074, 2001

49. Ruhul Amin AR, Senga T, Oo ML, Thant AA and Hamaguchi M: Secretion of matrix metalloproteinase- 9 by the proinflammatory cytokine, IL-1beta: a role for the dual signalling pathways, Akt and Erk. Genes Cells 8: 515-523, 2003.

50. Weng CJ, Chau CF, Hsieh YS, Yang SF and Yen GC: Lucidenic acid inhibits PMA-induced invasion of human hepatoma cells through inactivating MAPK/ERK signal transduction pathway and reducing binding activities of NF-kappaB and AP-1. Carcinogenesis 29: 147-156, 2008. 